\title{
Nursing Practice Environment and the Nurse Manager Intervention: Critical Review
}

\author{
Manuel Gomes $\mathrm{P}^{1 *}$ Lucas $\mathrm{P}^{2}$ \\ ${ }^{1}$ Graduate student in Nursing Management, Lisbon's Nursing School (ESEL), Portugal \\ ${ }^{2}$ Nursing Research, Innovation and Development Centre of Lisbon (CIDNUR), Nursing School of Lisbon, Portugal
}

\begin{abstract}
The Nursing Practice Environment (NPE) influences the quality of nursing care. Studies show far better outcomes for clients, with positive results on nursing teams' satisfaction and patients with improvements in the outcomes of patients and healthcare organizations. The Practice Environment Scale-Nursing Work Index (PES-NWI) is an instrument that measures the nursing practice environment and is currently the most used at a global scale. The analyzed article proves the instrumentsreliability in the assessment of the NPE, despite needing further investigation at a psychometric level; and its applicability in different care settings - Other countries besides the US. However, it is emphasized the need to carry out further experimental and longitudinal studies to potentially identify the mechanisms that influence the NPE.
\end{abstract}

Keywords: Nursing, Nurse Administrators, Quality of Care, Review, Work Environment

\section{Introduction}

"The Practice Environment Scale of the Nursing Work Index: An updated review and recommendations for use", by Swiger, Patrician, Miltner, Raju, Breckenridge-Sproat\& Loan, ${ }^{1}$ aims to provide an updated narrative review of the instrument "The Practice Environment Scale of the Nursing Work Index" as well as provide useful recommendations for its application in clinical settings by researchers and nurse managers. The article analyzes the instruments application in the modern times (in other workgroups besides Registered Nurses (RN) and in different countries); analyzes the association between PES-NWI and the different reported scores obtained in the studies, comparing them. Furthermore, they propose suggestions for future research projects on the subject matter.

The practice environment scale of the nursing work index

The PES-NWI was originally developed in 2002 by Eileen Lake, Professor at the University of Pennsylvania.

With the creation of this instrument, researchers and nurse managers can assess the NPE. The NPE is defined by the author as "the organizational characteristics of a work setting that facilitate or constrain professional nursing practice". Such characteristics could be variables like the relationship between nurses and physicians or the hierarchical status of nurses in the hospital. Amaral, Ferreira \& Lake ${ }^{3}$ argue that the PES-NWI is one of the most used instruments in the world to assess the NPE, being recognized and recommended by international entities like The National Quality Forum and the Joint Commission for Accreditation of Hospitals.The instrument comprises a total of thirty-one items organized in five categories/subscales, those being: "Nurse Participation in Hospital Affairs", "Nursing Foundations for Quality of Care", "Nurse Manager Ability, Leadership, and Support for Nurses", "Staffing and Resource Adequacy", “Collegial Nurse-Physician Relations". ${ }^{2-4}$

\section{Authors biographical information}

The article was written by a total of six authors, namely: Pauline Swiger, Patricia Patrician, Rebecca Milter, Dheeraj Raju, Sara Breckenridge-Sproat e Lori Loan. The first author, Pauline Swiger, has been a nurse for more than twenty years, initiating her work experience in the US army corps. Currently, she works as a Deputy

\begin{tabular}{|l|l|}
\hline \hline Quick Response Code: & FCorresponding author: Pedro Manuel Gomes. Graduate student in Nursing Management, \\
Lisbon's Nursing School (ESEL), Lisbon, Portugal \\
Received: 05 March, $2021 \quad$ Published: 17 March, 2021 \\
Citation: Manuel GP, Lucas P. Nursing Practice Environment and the Nurse Manager Interven- \\
tion: Critical Review. Trends Nur Health Care Res. 2021;1(1):1-5. DOI: \\
10.53902/TNHCR.2021.01.000503
\end{tabular}


Chief in the Center for Nursing Science and Clinical Inquiry and as a Clinical Inquiry in Landstuhl Regional Medical Center. The author holds a Bachelor of Science in Nursing by Viterbo University, awarded in 2000, a Master of Science in Nursing by the University of Texas San Antonio, awarded in 2013, and a PhD in Nursing Research at the University of Alabama at Birmingham, awarded in 2017.

The author has published ten articles in the past nine years, in four reputable journals, namely: "Research in Nursing \& Health", "Nursing Outlook", "Journal of Advanced Nursing" and the "International Journal of Nursing Studies".During those years, Swiger has also been involved in two projects "Psychometric Analysis of the PES-NWI" and "A program evaluation of the Army's nursing care delivery framework", the latter contributed to the publishing of 3 articles under her name. The author's main topics of interest and skills are described in her personal Research Gate page as being: Nursing Practice, Staffing, Nursing Workforce and Quality and Safety. Her institutional email is pswiger@uab.edu, and her personal Research Gate can be accessed through the following link: https:// www.researchgate.net/profile/Pauline_Swiger.

The last author of the article, Lori Loan, has been an Associate Professor for the past six years at the University of Alabama at Birmingham. The author holds a Bachelor of Nursing in Registered Nursing by Pacific Lutheran University, awarded in 1982, a Master of Nursing in Registered Nursing by the University of Washington, awarded in 1992, and a PhD in Nursing Science at the University of Washington, awarded in 2000. The author has published more than sixty-two articles in the past few decades, in numerous highly regarded journals, such as: "International Journal of Nursing Studies", "Nursing Outlook", "American Journal of Nursing", "American Journal of Infection Control" and the"Journal of Nursing Administration".

Presently, she is involved in two projects, "A program evaluation of the Army's nursing care delivery framework" and "Health Policy". Some of the author's main topics of interest and skills are described in her personal Research Gate page as being: Nursing Leadership, Nursing Management, Medical Science Nursing and Quality improvement. Her institutional email is loanl@uab.edu, and her personal Research Gate can be accessed through the following link: https://www.researchgate.net/profile/Lori_Loan.

\section{Analysis}

The article follows the structure of a narrative review, containing the following sections: Title, authors' information, addresses, keywords, abstract (where the objectives, study design, search sources, review methods, results and conclusion are presented), introduction (where concepts are defined and presented), search strategy, review findings (where the sample is presented containing information such as: methods utilized, reported reliability analysis, use across practice settings and countries, modifications and international use, scoring, the associations between the PES and outcomes), discussion and recommendations, limitations found, conclusion, funding, conflicts of interest, acknowledgements, appendix and references of the study.
A Prisma diagram was used to illustrate the sampling process. The final sample comprised forty-six articles. Additionally, a table (Table 1 in the original article) was used to depict the result of three studies comparing the PES-NWI mean scores of magnet, non-magnet, and emerging magnet hospitals. The appendix is referenced in the study; however, it can only be accessed online. The appendix contains further information about the sample- analyzing each article at a deeper level. Swiger's, et al. ${ }^{1}$ study is of upmost importance for nursing practice, bringing to the discussion an instrument that moves several concepts inherent to the nurse manager practice (Quality, Safety, Satisfaction, Human resource management, staffing, communication, etc.).

Nurse Managers play a key role in creating favorable/positive $\mathrm{NPE}^{5}$ and promoting the delivery of quality care.$^{6-7}$ They can also provide the necessary tools for the professional development of nurses and future managers. ${ }^{8}$ Nursing leadership plays a central role in quality of care, which involves four fundamental roles: facilitating effective continuous communication; strengthening intra and interprofessional relationships; creating and maintaining teams; and peer involvement. ${ }^{6}$ Nursing leadership directly influences the $\mathrm{NPE}^{7,9}$ and the quality of nursing care. ${ }^{6}$ Nurses as leaders, are fundamental to strengthen the communication with and within the team to further achieve goals, with the aim to provide quality care, promote patient's safety and innovate. ${ }^{6,10}$

\section{Selected references}

Swigers' et al. ${ }^{1}$ study possesses a large number of references, despite their temporal dispersion; it's oldest being from 1988 and the most recent one from 2017-being a self-reference to another study of hers. In fact, she self-references two of her previous works. All the referenced studies were published on scientific journals, except two-one being a reference to "google scholar" and another one to "scopus" where she got the number of citations in the mentioned study. The article references key authors on the subject matter, such as: Aiken, L; Kutney-Lee, A. and Lake, E.

\section{Analysis per section}

In order to extract and analyze the largest amount of information possible, we'll proceed to analyze the article section by section.

\section{a. Introduction}

The introduction schematically presents the existing evidence on the theme and what the article adds to the topic. They present a theoretical framework about the instrument, explaining the definition by the original author and a brief explanation about the existing evidence on the instrument (higher scores in PES-NWI are associated with better outcomes, namely improving the quality of care, less therapeutic errors, fewer falls, as well as better reported experiences by patients. ${ }^{11-16}$ Moreover, they briefly explain the five categories comprising the PES-NWI(Nurse Participation in Hospital Affairs; Nursing Foundations of Quality Care; Nurse Manager, Leadership, and Support of Nurses; Staffing and Resource Adequacy; and Collegial Nurse-Physician Relations). ${ }^{2}$ They conclude the introduction by presenting the objectives already highlighted in the abstract, as well as other goals that they intend to grasp in the 
study, specifically: to identify current scores of the instrument in the literature and its meaning/effects in practice; assess the investigative progress regarding the recommendations proposed in the original article; identify changes made to the instrument and variations in scoring; and evaluate the applicability of the instrument in populations other than RNs.

\section{b. Search strategy}

Pubmed, CINAHL and Embase were searched using the words "Practice Environment Scale of the Nursing Work Index" and "PESNWI", culminating in an initial sample of two hundred studies. Subsequently, inclusion criteria were applied (articles written in English that focused on the relationship between PES-NWI and the reported scores in specific groups (units, wards, etc.). Although not directly explicit in this section, we can see in the developed PRISMA diagram that only studies published between 2010 and 2016 were included. After abstract screening twenty-seven studies were excluded - review articles, translations of PES-NWI or academic dissertations. From the eighty-three articles retrieved, eleven were excluded after full text screen and twenty-six were excluded during data extraction. ${ }^{1}$

\section{c. Review findings}

The final sample containedforty-six studies, from which twenty-five were published in peer-reviewed journals- nearly half the sample (43\%) was published in international journals. ${ }^{1}$

\section{d. Study designs and samples}

In this section, the sample's studies designs were presented as well as their respective samples characteristics.

In the analyzed sample of forty-six studies, 93\% were cross-sectional studies, one was a longitudinal study, and the otherwas an experimental one. Twenty-five articles collected data from primary sources and twenty-two from secondary ones (RN4Cast, Vermont Oxford Network database, Multistate nursing care and patient safety survey and the swiss nursing home human resources project). The samples in the respective studies ranged from 133 to 33,845 nurses. $59 \%$ of the articles used only answers given by RN's. In the remaining $41 \%$, responses from other types of nurses were accepted, such as: "advanced practice nurses, licensed practical/vocational nurses (LPN/LVN), enrolled nurses (EN-Australia), certified nurse's assistants (CNA), nurse's aides/technicians, and primary, junior, and senior nurses (China)". ${ }^{1}$ Only a few studies included nurse managers/leaders. A study that included nurse managers, concluded that they overestimated the NPE compared to other nurses, with the exception of items related to staffing and adequate resources. Many studies only included nurses with three, six or even twelve months of experience. The exclusion of nurses with little experience in the workplace, allows a more correct assessment of PES-NWI. ${ }^{1}$

\section{Methods}

According Warshawsky\& Havens ${ }^{17}$ the use of a multilevel analysis better reflects the NPE and the instrument's performance in its five different categories. One third of the selected studies used this type of analysis. One study used a mixed descriptive method.

\section{Reported realiabilty analisys}

To assess the data reliability, most of the studies in sample applied the Cronbach's alpha coefficient. Seven studies only reported the Cronbach's alpha coefficient from other studies-some of which were older studies with samples from different geographical settings. Thirty-seven studies applied the Cronbach's alpha coefficient or another way to measure the instrument reliability. Two studies did not assess its reliability. ${ }^{1}$

\section{Use across practice settings and countries}

In this section the authors explore the geographical application of PES-NWI in different care settings. PES-NWI was applied in several countries-a total of 28 countries in the included articles. Most of the studies assessed a context of acute healthcare, namely intensive care units (adults, neonatal and pediatric), med/surgeries, oncology, orthopedics, psychiatry, gastroenterology, emergency wards and the operating room. One of the studies validates the use of PES-NWI in outpatient/ambulatory care in the US and Spain. ${ }^{1}$

\section{Modifications and international use}

According to the analyzed studies, the instrument has been changed to adapt it to each of the different care settings or countries of origin, changing for example the titles given to nurses (RNs, LPN, etc.), or adapting certain items to the countries nursing culture practice (e.g., the removal of nursing diagnoses in four Australian studies or adequacy of the words used in an oncology care setting). ${ }^{1}$

\section{Scoring}

PES-NWI uses a 4-point Likert-type scale that allows the user to identify elements that promote or inhibit the provision of quality care. The higher the score, the better the NPE. Items with a value greater then 2.5 are deemed favorable. Lake \& Friese ${ }^{18}$ categorize NPE in three different levels: poor, mixed, and favorable. Favorable NPE when it receives an average of 2.5 in four of the five subscales, mixed when it receives 2.5 in two or three of the five subscales and poor when it receives 2.5 in one or none of the five mentioned subscales. ${ }^{1,18}$ Some of the analyzed articles made an adjustment to the proposed Likert scale, as they used another analysis instrument, maintaining the consistency of the data.

\section{Reported PES-NWI scores}

In this section the authors scrutinize the reported scores of the studies included in the sample. Sixteen of the studies presented mean scores ranging from 2.3 to 3.07. Reported scores analyzed confirmed Warshawsky \& Haven ${ }^{17}$ evidence-lowest scores are related to Staffing and adequacy of resources.

\section{The associations between the PES and the outcomes}

Of the forty-six retrieved articles, the majority associated PESNWI with patient, nurse, and organizational outcomes. Nonetheless, twenty-one studies only reported the significance of the associations, but showed no evidence to support the strength of the described associations. ${ }^{1}$ 


\section{Nursing outcomes}

Twenty-four of the studies investigated nursing outcomes (e.g., job satisfaction, intent to leave, burnout, etc.), and most studies found a significant relationship between nurse outcomes of interest and one of the PES-NWI subscales.Several studies showed a negative association with burnout, intent to leave and job satisfaction. ${ }^{1}$

\section{Patient outcomes}

Fourteen studies investigated patient outcomes (e.g. care satisfaction, medication errors, etc.). The results in some of the studies were inconclusive, nevertheless some studies showed that a favorable NPE indicates less medication errors and better evaluation of the nursing care provided.

\section{Organizational variables}

Eight studies investigated organizational outcomes (e.g. safe environment, reporting of errors, etc.). Three studies revealed that a favorableNPE results in greater error reporting and organizational safety. ${ }^{1}$

\section{Discussion and recommendations}

In this section the authors discuss the results and provide recommendations for nurse managers and researchers.

\section{Design and methods}

The authors suggest the need to conduct more longitudinal and experimental studies to generate stronger evidence related to the NPE. Additionally, they criticize the Cronbach's alpha value used by some of authors included in the sample for using Lakes original value-as the value varies according to the population. ${ }^{1}$

\section{Sampling}

It is recommended to use PES-NWI only in nurses who provide direct care to patients, given the variance found when applied to nurse managers.It is also questioned the reliability of the minimum set answers defined by a unit/hospital-5 to a unit and 10 to a hospital, requiring further investigation to determine if they are sufficient.The instrument was developed being applied only to RNs from Magnet hospitals; it is highlighted the need to investigate its reliability in other nurses other than RNs. The authors also emphasize the need to update the instrument to the current reality of nursing practice, given the few changes made since its creation. ${ }^{1}$

\section{Use across practice settings, modifications and scoring}

PES-NWI has been applied to a wide range of clinical settings (ambulatory care, clinics, etc.), maintaining its reliability assessing the NPE. In this section the authors compare the different versions found of PES-NWI and the scoring differences in each of the versions.

\section{Reporting Associations}

It is reported a high probability of type 1 errors, given the amount of tests to compute when statistically analyzing the outcomes. Moreover, Swiger, et al. mention Lakes recent meta-analysis $^{19}$ results that highlights the importance of the NPE relation to nurse and patients'outcomes-higher care satisfaction, lower adverse events and higherquality of care reported by nurses.

\section{Limitations}

The search strategy was identified as a limitation given its rather narrow inclusion criteria and low amount of words/terms used. Regardless, they identify that the use of the terms "Nursing Practice Environment" and "Care Environment" would broaden their sample to nearly five thousand articles. The omission of the mentioned articles may have led to the exclusion relevant studies, narrowing the amount of studies that assess the association of PES-NWI with patient, nurse and organizational outcomes.

\section{Conclusion}

I. PES-NWI remains a common and reliable way of evaluating the NPE, however the instrument still needs further research at a psychometric level.

II. There's still a need to conduct more longitudinal and experimental studies in order to identify practical mechanisms/ interventions that influence the NPE. ${ }^{1}$

\section{Appendix A. Supplementary Data}

The original appendix can be accessed through Science Direct (Elsevier's platform) providing further information about each of the articles included in the sample, namely: Name of the authors, year of publishing, sample, where it was conducted (country), outcomes, etc. ${ }^{1}$.

\section{References}

The International Journal of Nursing Studies reference style was used in the original article.

\section{Results and Discussion}

The results obtained by Swiger, et al. ${ }^{1}$, are relevant to the modern nursing practice and prove that PES-NWI can be applied in different care settings and in different countries besides the US. Both the narrative review and PES-NWI can be applied to the current Portuguese nursing care practice. The instrument has been validated and translated to the Portuguese population proving its applicability. Recently it has been validated with a good fit to the 5-factor model ${ }^{4}$-this was the largest study on the NPE ever carried out in Portugal. The applicability of this instrument is of upmost relevance in a clinical setting, allowing nurse managers and researchers to evaluate the NPE and the inherent variables that promote or inhibit it. The instrument in question is a remarkable management tool, scoping and targeting weaknesses and strengths of the NPE and thus allowing the managers to prepare interventions aimed at making it favorable culminating in the promotion of nursing care quality.

\section{e. Suggestions for future works}

For future works, the authors propose the conduction of longitudinal and experimental studies in order to identify the mechanisms on how the NPE impacts the nursing, people and organizational outcomes; doing this, we'll obtain further evidence on the subject and we'll be able to identify concrete interventions to enhance the NPE. In addition, they propose the conduction of interventional studies to assess if higher NPE scores reflect better 
outcomes for nurses, patients, and organizations.We believe that the proposed studies are relevant to highlight concrete interventions that nurse managers and researchers can apply to enhance the NPE; and at the same time validate its practical relevance - if better or worse NPE scores are associated with a higher quality of nursing care.

\section{Conclusion}

Swiger's, et al. ${ }^{1}$ study is of tremendous relevance regarding the validation and applicability of PES-NWI worldwide; evaluating the evidence covered in studies of twenty-six countries on different professional nurse groups; but also evaluating the reported scores, scoring methods and association with the outcomes.

The authors of the mentioned article are all carriers of great knowledge on the subject; all of six possessing PhDs in Nursing with several publications in respectable international journals; some of them with great teaching experience at an academic level on the said subject proving and highlighting their undeniable impact in the area of studies.

The results obtained bring developments in the study of the instrument, and still leave pertinent recommendations for future studies; aiming to improve/update it.

Reading and analyzing the article, we would also like to make some suggestions for future works on the topic:

1) A study where they assesson how the cultural-socio-economic impact of a country enhances or diminishes the reported outcome scores obtained in hospitals, for example: in developing countries, with low investment by health ministers - how are the reported scores affected? Specifically, in the adequacy of material and human resources.

2) A study where they apply PES-NWI in nurse-lead units, specifically in primary care settings and how does it behave at a psychometric level.

We recommend the article by Swiger, et al. ${ }^{1}$, for the excellent content shown in the study, as well as for all the knowledge mobilized in it, which reveals itself of great depth, both in nursing and nursing research, being revealed by the authors in the way they criticize, discuss, and argue the results obtained in the different articles and how they could be improved.

\section{Funding}

None.

\section{Conflicts of interest}

Author declares that there is no conflict of interest.

\section{Acknowledgments}

In this section the authors further acknowledge the mentioned entities for the funding, aswell as the School of Nursing, University of Alabama at Birminghamfor the support provided throughout the manuscript's elaboration.1 The study was funded by the U.S. Army Nurse Corps, Long Term Health Education and Training grant, TriService, Nursing Research Program, and by the Uniformed Services University of the Health Sciences.

\section{References}

1. Swiger PA, Patrician PA, Miltner RSS, et al. The Practice Environment Scale of the Nursing Work Index: An updated review and recommendations for use. Int J Nurs Stud. 2017;74:76-84.

2. Lake ET. Development of the practice environment scale of the nursing work index. Res Nurs Health. 2002;25(3):176-188.

3. Amaral A, Ferreira PL, Lake E. Validation of the Practice Environment Scale of the Nursing Work Index (PES-NWI) for the Portuguese nurse population. Int J Car Sci. 2012;5:280-288.

4. Almeida S, Nascimento A, Lucas PB, et al. Rn4cast study in Portugal: Validation of the Portuguese version of the practice environment scale of the nursing work index. Aquichan. 2020;20(3):1-10.

5. Alves D, Guirardello E. Nursing work environment, patient safety and quality of care in pediatric hospital. Rev GauchaEnferm. 2016;37(2):58817.

6. Carvalho MC, Lucas PR. The effectiveness of the clinical nurse leader practice-systematic review. Millenium. 2020;2(11):57-64.

7. Lucas PRMB, Nunes EMGT. Nursing practice environment in Primary Health Care: a scoping review. Rev Bras Enferm. 2020;73(6):e20190479.

8. Gea-Caballero V, Castro-Sánchez E, Júarez-Vela R, et al. Elementos esenciales de los entornos profesionalesenfermeros en Atención Primaria y su influencia en la calidad del cuidado. EnfermClín. 2018;28(1):27-35.

9. Weber E, Ward J, Walsh T. Nurse leader competencies: A toolkit for success. Nurs Manage. 2015;46(12):47-50.

10. Nunes EMGT, Gaspar MFM. Leadership in nursing and patient satisfaction in hospital context. Rev GaúchaEnferm. 2016;37(2):e55726.

11. Friese CR. Nurse practice environments and outcomes: Implications for oncology nursing. Oncol Nurs Forum. 2005;32(4):765-772.

12. Kutney-Lee A, Lake ET, Aiken LH. Development of the hospital nurse surveillance capacity profile. Res Nurs Health. 2009;32(2):217-228.

13. Spence Laschinger HK, Leiter MP. The impact of nursing work environments on patient safety outcomes: the mediating role of burnout/engagement. J Nurs Adm. 2006;36(5):259-267.

14. Manojlovich M, De Cicco B. Healthy Work Environments, NursePhysician Communication, and Patients' Outcomes. Ame J Crit Care. 2007;16(6):536-543.

15. McCusker J, Dendukuri N, Cardinal L, et al. Nursing work environment and quality of care: Differences between units at the same hospital. Int J Health Care Qual Assur. 2004;17(6):313-322.

16. Patrician PA, Shang J, Lake ET. Organizational determinants of work outcomes and quality care ratings among army medical department registered nurses. Res Nurs Health. 2010;33(2):99-110.

17. Warshawsky NE, Havens DS. Global use of the practice environment Scale of the Nursing Work Index. Nurs Res. 2011;60(1):17-31.

18. Lake ET, Friese CR. Variations in Nursing Practice Environments Relation to Staffing and Hospital Characteristics. Nurs Res. 2006;55(1):1-9.

19. Lake ET, Sanders J, Rui D, et al. A meta-analysis of the associations between the nursing work environment in hospitals and five sets of outcomes. Paper presented at the academy Health, Boston, MA. 2016. 